\title{
Formation of a novel shaped bunch to enhance transformer ratio in collinear wakefield accelerators
}

\author{
B. Jiang, ${ }^{1,2}$ C. Jing, ${ }^{1,3}$ P. Schoessow, ${ }^{3}$ J. Power, ${ }^{1}$ and W. Gai ${ }^{1}$ \\ ${ }^{1}$ Argonne National Laboratory, 9700 South Cass Avenue, Argonne, Illinois 60439, USA \\ ${ }^{2}$ Shanghai Institute of Applied Physics, CAS, Shanghai 201800, China \\ ${ }^{3}$ Euclid Techlabs, 5900 Harper Road, Solon, Ohio 44139, USA \\ (Received 18 November 2011; published 10 January 2012)
}

\begin{abstract}
The transformer ratio, characterizing the ratio of maximum accelerating field behind the drive bunch to the maximum decelerating field inside the drive bunch, is one of the key parameters for characterizing the performance of collinear wakefield accelerators. In this paper the use of electron drive bunches possessing a particular temporal profile (the double-triangular bunch) is shown to significantly increase the transformer ratio beyond 2, the limit for a symmetric bunch. The double-triangular bunch is generated using the emittance exchange technique [P. Emma et al., Phys. Rev. ST Accel. Beams 9, 100702 (2006)]. Complete beam simulations have been performed for a collinear wakefield acceleration experiment planned at the Argonne Wakefield Accelerator facility including the effects of the physical emittance increase of the bunch in the wakefield device. A transformer ratio of 6.5 and gradient of $20 \mathrm{MV} / \mathrm{m}$ is expected in this proof-of-principle experiment for a $3 \mathrm{nC}$ double-triangular bunch traversing a $10 \mathrm{~cm}$ long, $200 \mathrm{GHz}$ quartz based dielectric wakefield accelerator structure.
\end{abstract}

DOI: 10.1103/PhysRevSTAB.15.011301

PACS numbers: 29.20.Ej

\section{INTRODUCTION}

Future high energy colliders as well as future free electron laser light sources will require high accelerating gradients, making wakefield acceleration a very attractive candidate technology [1-4]. The underlying principle of wakefield acceleration is that a short, relatively low energy, but high current drive beam creates high accelerating fields in a wakefield device, which can then be used to accelerate a trailing beam to high energy. Two figures of merit in wakefield accelerators are the transformer ratio $R$ and the accelerating gradient. $R$ is defined as the ratio of the maximum accelerating field behind the drive bunch to the maximum decelerating field inside the drive bunch, and characterizes the energy transfer efficiency from the drive beam to the accelerated (witness) beam. This is ultimately related to the overall efficiency (under some constraints, e.g., a fixed beam loading, the same wakefield structure, etc.). Obviously a high value of the transformer ratio is preferred. Meanwhile the presence of a high accelerating gradient is always attractive because it will reduce the main linac length which is the primary determining factor for the facility size.

For a finite length, longitudinally symmetric bunch, the transformer ratio $R$ has an ordinary upper limit of 2 [5]. The general solution to increase the transformer ratio $R$ above 2 in a collinear wakefield accelerator is to use a

Published by the American Physical Society under the terms of the Creative Commons Attribution 3.0 License. Further distribution of this work must maintain attribution to the author(s) and the published article's title, journal citation, and DOI. bunch or bunch train which has an asymmetric temporal profile, for example, a bunch with ramped charge (triangular beam and some variations) [5], and a ramped bunch train (a train of electron bunches with specific ramped-up charge ratio but equal time separation, i.e., an amplitude modulated bunch train) [6,7]. The ramped bunch train technique has been experimentally demonstrated at Argonne Wakefield Accelerator (AWA) facility [7]. $R \sim 3.4$ was recently obtained [8]. However, it appears to be a great challenge to achieve a higher $R$ using a longer bunch train due to the tight requirements on the space and charge ratio among bunches in the train [8]. These technical challenges can be greatly reduced in an approach using a triangular shaped drive bunch. Techniques to produce such a shaped bunch have been significantly improved (although challenges still remain) since this approach to enhance the transformer ratio of a plasma wakefield accelerator using a triangular bunch was first raised [9]. Methods that have been explored to generate a triangular bunch include the compression of a time-energy chirped bunch with an anisochronous dogleg beam line [10]; temporal shaping of the drive-laser intensity in a photoinjector source [11]; manipulating the longitudinal phase space nonlinearities induced from linacs operating at different frequencies [12]; and a mask to select a transverse shape then switch it to longitudinal space with an emittance exchange (EEX) beam line [13].

It should be pointed out that the concept of transformer ratio is much less important in a two-beam-accelerator (TBA) scheme, where the wakefield energy of the drive bunch is extracted and coupled into another external powered accelerator. Because the impedance of two structures, 
wakefield power extractor and accelerator, can be made drastically different (the extractor is low impedance and the accelerator is high impedance), the transformer ratio is usually very high. Taking the CLIC $3-\mathrm{TeV}$ design as an example [14], the main beam gains $62.5 \mathrm{GeV}$ in one stage while the drive beam energy is reduced from $2.38 \mathrm{GeV}$ to $240 \mathrm{MeV}$, giving a transformer ratio of 31.25 . However, the number of structures in the TBA is doubled compared to the collinear scheme even though we assume they have the same average gradient. Moreover, the gradient of the accelerators in the TBA scheme seems to be limited to $\sim 120 \mathrm{MV} / \mathrm{m}$, particularly for conventional metallic disk-loaded accelerating structures operated in the microwave regime [15]. A coaxial wakefield device in principle can combine the advantages of collinear and TBA schemes $[16,17]$, but it also faces great challenges in drive beam formation, structure construction, beam transport, etc.

In this paper, we first analyze the transformer ratio of an asymmetric bunch and introduce a new longitudinal charge distribution we refer to as the double-triangle (DT) bunch which can produce higher $R$ than a single triangular bunch. In Sec. III, a complete numerical simulation of doubletriangular bunch formation in an EEX beam line currently under construction at the AWA is presented, and in Sec. IV, a quartz based dielectric wakefield accelerator (DWA) is designed to perform the enhanced $R$ wakefield experiment. Beam dynamics of the DT bunch traversing the DWA is discussed in Sec. V. The paper concludes with a discussion of planned future research directions.

\section{LONGITUDINAL WAKEFIELD FROM A DT BUNCH}

For a structure based wakefield accelerator, the longitudinal wake function can be simplified to [18]

$$
W_{z}(t)=\sum_{i} 2 k_{i} \cos \left(\omega_{i} t\right),
$$

where $k_{i}$, and $\omega_{i}$ are respectively the loss factor and the resonant frequency of the wake function for each mode. The wake potential of the bunch is the convolution integral of the wake function and the current distribution $I(t)$ :

$$
V(t)=-\int_{-\infty}^{t} I(\tau) W_{z}(t-\tau) d \tau .
$$

The loss factor is defined as $k_{i}=\omega_{i}[R / Q]_{i} / 4$, where $[R / Q]_{i}$ is the figure of merit of an accelerating structure for each mode and depends only on the geometry of the structure. In general, a smaller beam aperture will result in higher frequency and $[R / Q]$ of the fundamental mode, which in turn lead to a higher loss factor for accelerating structures. Contributions from the high order modes (HOMs), despite their high frequencies, will drop rapidly because of the low $R / Q$ (one exception is a multimode structure that is purposely designed to allow HOMs to contribute a larger portion of the wakefield [19]). Equation (2) reveals the fact that, for a given charge, the peak value of wakefield can be greatly increased if the drive bunch is compressed both longitudinally and transversely (with the transverse size of the wakefield structure reduced accordingly). This can be illustrated by two experiments: (1) in [20], $100 \mathrm{MV} / \mathrm{m}$ was achieved in a microwave dielectric wakefield device $(3.8 \mathrm{~mm}$ beam aperture and $\sim 5 \mathrm{kA}$ peak current); (2) in [21], $>5 \mathrm{GV} / \mathrm{m}$ was reached in a $\mathrm{THz}$ dielectric wakefield device $(0.1 \mathrm{~mm}$ beam aperture and $\sim 10 \mathrm{kA}$ peak current). However, in both experiments a Gaussian bunch (temporal profile of the bunch is a Gaussian distribution) was used so that the transformer ratio in these experiments could not exceed 2, which means the energy gained by the witness bunch is at the cost of the drive bunch losing at least half of that amount in the collinear wakefield acceleration scheme.

In order to enhance the transformer ratio, the shaped drive bunch or bunch train is the only approach if the drive and witness bunch experience the same structure impedance. One scenario that has attracted broad attention is the triangular bunch. Consider a triangular bunch which has a current density distribution:

$$
I(t)=I_{0} \omega_{0} t \text { for } 0<t<T \text { and } I(t)=0 \text { otherwise. }
$$

Here $I_{0}$ is a constant, and $\omega_{0}$ is the frequency of the lowest mode of the wakefield device (for simplicity, we consider a single mode wakefield structure for now). The decelerating field inside the drive bunch is

$$
\begin{aligned}
V^{-}(t) & =-\int_{-\infty}^{t} 2 k I_{0} \omega_{0} \tau \cos \left[\omega_{0}(t-\tau)\right] d \tau \\
& =-\frac{2 k I_{0}}{\omega_{0}}\left(\cos \omega_{0} t-1\right)
\end{aligned}
$$

which shows that the particles lose different energies in the wakefield device depending on their position in the bunch. Particles present at $t=\pi N / 2 \omega_{0}$ do not lose energy at all so that they do not contribute to the wakefield.

Now let us slightly change the bunch current distribution to the so-called "double-triangular distribution," which has a current profile described by

$$
I(t)= \begin{cases}I_{0} \omega t & 0<t<\frac{\pi}{2 \omega} \\ I_{0} \omega t-I_{0} & \frac{\pi}{2 \omega}<t<T .\end{cases}
$$

Using Eq. (2) we have the decelerating field inside the bunch:

$$
V^{-}(t)= \begin{cases}-\frac{2 k I_{0}}{\omega}[1-\cos (\omega t)] & 0<t<\frac{\pi}{2 \omega} \\ -\frac{2 k I_{0}}{\omega} & \frac{\pi}{2 \omega}<t<T .\end{cases}
$$

The wakefields generated by a DT bunch and a single triangular (ST) bunch are plotted in Fig. 1. For comparison, both bunches are defined to pass through the same single frequency wakefield device. They have the same bunch length and their current profiles follow Eqs. (3) and (5), respectively, and are normalized to unit charge. Figure 1 clearly shows that the energy loss inside the DT bunch is 

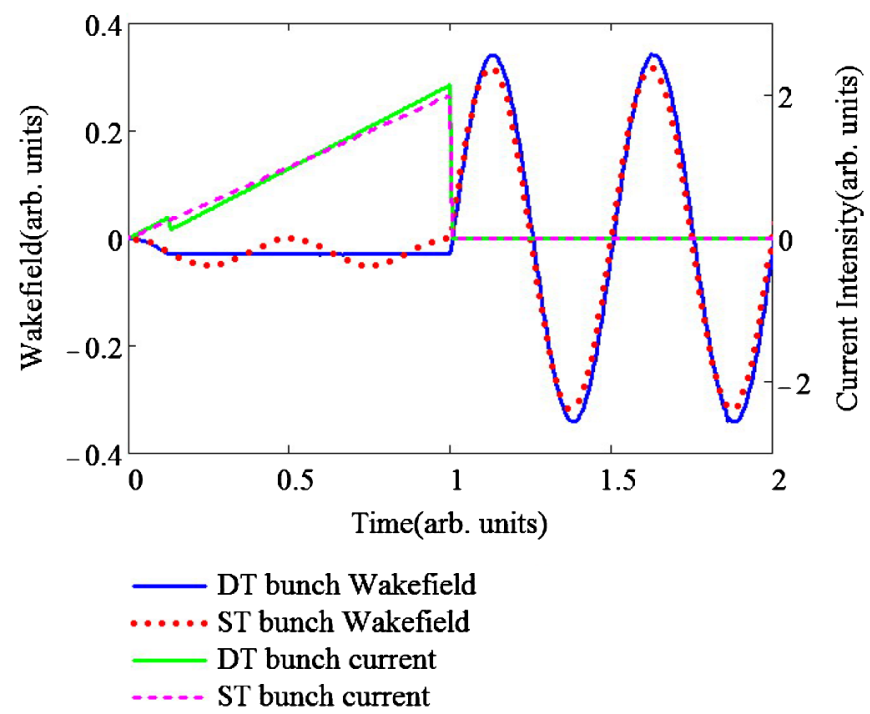

FIG. 1. Wakefields for the ideal DT and ST bunches traversing a single mode wakefield device.

nearly constant so that its transformer ratio is higher and the induced energy spread is less. The transformer ratio in an ideal DT bunch [using Eq. (2)] is

$$
R=\sqrt{1+(\omega T-1)^{2}}
$$

nearly twice the $R$ for a ST bunch. The example in Fig. 1 shows $R=11.6$ for the DT bunch and $R=6.3$ for the ST bunch. It might be worth pointing out that, comparing to the "doorstep" mentioned in [9], $R$ of the DT bunch is very similar; but the DT bunch is easier to create experimentally because only one edge of the beam profile needs to be steeply cutoff.

\section{Influence of the slowly dropping tail}

In the wakefield analysis, the current density distribution of the triangular bunch or its variations is assumed to have a steep cutoff edge at the bunch tail. In reality space charge effects, coherent synchrotron radiation, and energy divergence of the bunch will diffuse the particles in phase space and cause some difficulties in generating a sharp edged bunch in experiments. To address this issue, consider the current distribution shown in Fig. 2. For example, if $T_{2}=$ $2 T_{1}$, the distribution is close enough to symmetric that the transformer ratio will decrease to around 2. To minimize this tail effect, we require that all the particles at the tail (particles in $T_{1}<t<T_{2}$ ) slip away from the accelerating phase of the wakefield, i.e., they all lose energy and contribute to the wakefield behind bunch. Meanwhile, the decelerating field within the tail has to be maintained to be no greater than that from the front of bunch. These conditions can be expressed as follows:

$$
V^{-}\left(0<t<T_{2}\right)<0 \quad\left|V^{-}\left(0<t<T_{1}\right)\right| \geq\left|V^{-}\left(T_{1}<t<T_{2}\right)\right| .
$$

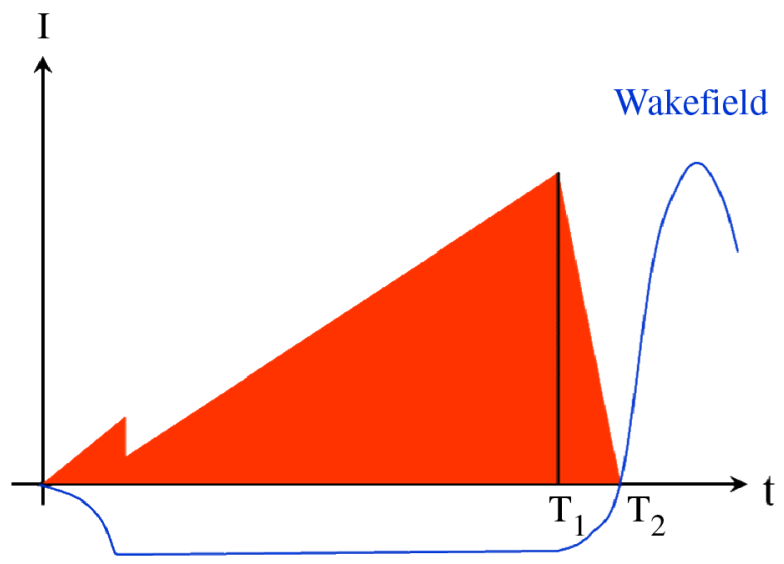

FIG. 2. Illustration of the double-triangular bunch with a dropping tail and its wakefield.

With the assumption of a linear tail from $T_{1}$ to $T_{2}$, the condition to maintain the nondegraded transformer ratio is

$$
\cos (\omega \Delta T) \geq \frac{\omega T_{1}-1}{\omega T_{1}-1+\omega \Delta T},
$$

where $\Delta T=T_{2}-T_{1}$. For example, if $\omega T_{1}=4 \pi$, then $\omega \Delta T<0.054 \pi$ should be satisfied to obtain the maximum transformer ratio of $3.7 \pi$.

\section{DT GENERATION USING THE EEX BEAM LINE}

The reason to choose the double-triangular bunch is that it is able to enhance the transformer ratio more than a single triangular bunch and can be implemented using a new approach: bunch shaping via an emittance exchange (EEX) beam line [22]. The EEX beam line, in a general sense, can be treated as a transport matrix between the transverse and longitudinal phase space, in such a way that one can manipulate the beam for different needs. For example, if a specific transverse mask is applied at the entrance of the EEX beam line then a longitudinal distribution can be shaped accordingly at the beam line exit.

The physics behind of EEX beam line is presented in detail in Refs. [22-24]. Here we briefly introduce the concept in a simplified manner. Figure 3 shows a sketch of a typical EEX beam line, which is composed of two dispersive sections (the so-called doglegs) separated by an rf deflecting cavity. The transport matrix of the dogleg $M_{D}$

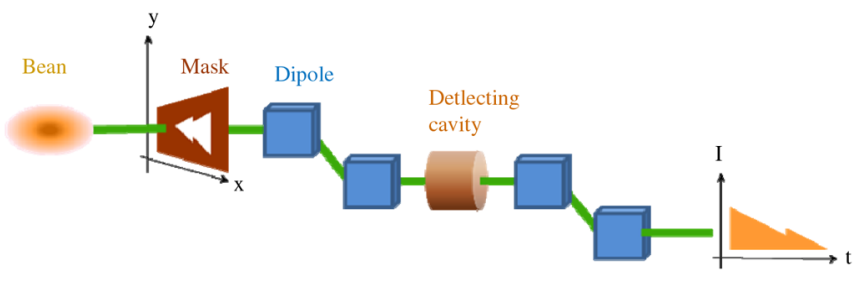

FIG. 3. Simplified sketch of a EEX beam line. 
can be expressed as [considering only the horizontal $(x)$ and longitudinal $(z)$ planes] [24]

$$
M_{D}(\eta, \xi, L)=\left[\begin{array}{llll}
1 & L & 0 & \eta \\
0 & 1 & 0 & 0 \\
0 & \eta & 1 & \xi \\
0 & 0 & 0 & 1
\end{array}\right],
$$

where $L, \eta$, and $\xi$ are the length, dispersion, and momentum compaction of the dogleg, respectively. The rf deflecting cavity, which operates on a $\mathrm{TM}_{110}$-like mode, delivers a longitudinal kick to the particle with strength in proportion to the distance from the axis. The transport matrix $M_{c}$ of the deflecting cavity in the thick lens approximation can be written as follows [24]:

$$
M_{C}(k)=\left[\begin{array}{cccc}
1 & L_{c} & k L_{c} / 2 & 0 \\
0 & 1 & k & 0 \\
0 & 0 & 1 & 0 \\
k & k L_{c} / 2 & k^{2} L_{c} / 4 & 1
\end{array}\right],
$$

where $L_{c}$ is the length of the cavity, $k=V_{0} /\left(a E_{0}\right)$ is the cavity strength parameter, $V_{0}$ is the cavity voltage, $a=$ $\lambda /(2 \pi), \lambda$ is the wavelength of the $\mathrm{TM}_{110}$-like mode, and $E_{0}$ is the beam energy. For a complete exchange, the dispersion $\eta$ and the cavity strength $k$ should satisfy [23]

$$
1+k \eta=0 .
$$

Using Eqs. (10)-(12), the coordinates of a particle at the exit of the EEX section can be obtained:

$$
\begin{aligned}
{\left[\begin{array}{c}
x_{2} \\
x_{2}{ }^{\prime} \\
z_{2} \\
\delta_{2}
\end{array}\right] } \\
=\left[\begin{array}{cccc}
0 & L_{c} / 4 & \left(\frac{L_{c}}{4}+L\right) k & -\frac{1}{k}+\left(\frac{L_{c}}{4}+L\right) k \xi \\
0 & 0 & k & k \xi \\
k \xi & -\frac{1}{k}+\left(\frac{L_{c}}{4}+L\right) k \xi & \frac{L_{c}{ }^{2} \xi}{4} & \frac{L_{c} k^{2} \xi^{2}}{4} \\
k & \left(\frac{L_{c}}{4}+L\right) k & \frac{L_{c} k^{2}}{4} & \frac{L_{c} k^{2} \xi}{4}
\end{array}\right] \\
\\
\times\left[\begin{array}{c}
x_{1} \\
x_{1}{ }^{\prime} \\
z_{1} \\
\delta_{1}
\end{array}\right],
\end{aligned}
$$

where $\left(x_{i}, x_{i}{ }^{\prime}, z_{i}, \delta_{i}\right)$ denotes coordinates of the particle at horizontal and longitudinal phase planes, and the subscripts 1 and 2 refer to coordinates before and after the EEX section, respectively. For a thin cavity $\left(L_{c}=0\right)$, the transform matrix in Eq. (13) will reduce to a block antidiagonal form so that the transverse and longitudinal coordinates are directly mapped to each other. Typically, $L$ is about a meter, $L_{c}$ is about $0.1 \mathrm{~m}, \xi$ is about several millimeters, and $k$ is usually less than 10 .

DT bunch generation using the EEX technique and a subsequent transformer ratio enhancement experiment are planned for the Argonne Wakefield Accelerator (AWA) facility. The AWA facility is currently undergoing a major upgrade [25]. A new $75 \mathrm{MeV}$ beam line is under construction, which will provide gigawatt level beam power as a driver for both two-beam accelerator and collinear wakefield accelerator experiments. Meanwhile, in order to balance the capability, flexibility, and functionality of this research facility, a new beam line switchyard will be constructed to allow multiple experiments: collinear wakefield acceleration; rf power generation and two-beam acceleration; EEX, etc. This flexible beam line switchyard will allow a quicker and more efficient transition among several concurrent experimental setups.

A start to end simulation of the double-triangular bunch generation, covering the AWA photocathode to the entrance of wakefield device, was performed using PARMELA (including space charge effects) [26]. In-house MATLAB routines were written to handle the particle input/ output at the mask and to apply the deflecting cavity thick lens matrix. The AWA beam is generated from an L-band UV photocathode gun and then accelerated to $75 \mathrm{MeV}$ through six linac tanks. A $100 \mathrm{nC}$ bunch train can be generated with the help of a high quantum efficiency (CsTe) cathode and a $15 \mathrm{~mJ}$ (before being split into micropulses) UV laser. The AWA beam parameters used in the simulation are listed in Table I. The existing AWA EEX beam line settings are listed in Table II. As a short bunch is needed to obtain a high wakefield gradient, the EEX is performed at $75 \mathrm{MeV}$ because space charge effects are small at this level and the unnormalized transverse

TABLE I. AWA injected beam parameters used in the simulation.

\begin{tabular}{lc}
\hline \hline Beam energy & $75 \mathrm{MeV}$ \\
Bunch charge (before the mask) & $10 \mathrm{nC}$ \\
Photon gun laser size, radius & $3.5 \mathrm{~mm}$ \\
Launch gun phase & $22^{\circ}$ \\
Transverse emittance (normalized) & $29.3 \mathrm{~mm} \mathrm{mrad}$ \\
Energy divergence (correlated) & $507 \mathrm{keV}$ \\
Energy divergence (uncorrelated) & $15 \mathrm{keV}$ \\
Bunch length & $1 \mathrm{~mm}$ \\
\hline \hline
\end{tabular}

TABLE II. EEX beam line settings.

\begin{tabular}{lc}
\hline \hline Bunch charge (after mask) & $3.72 \mathrm{nC}$ \\
Dipole length & $0.327 \mathrm{~m}$ \\
Bending angle & $22^{\circ}$ \\
Drift space between bends & $1.0 \mathrm{~m}$ \\
Deflecting cavity strength, $k$ & 3.3 \\
Normalized emittance $X$ (after EEX) & $74.6 \mathrm{~mm} \mathrm{mrad}$ \\
\hline \hline
\end{tabular}



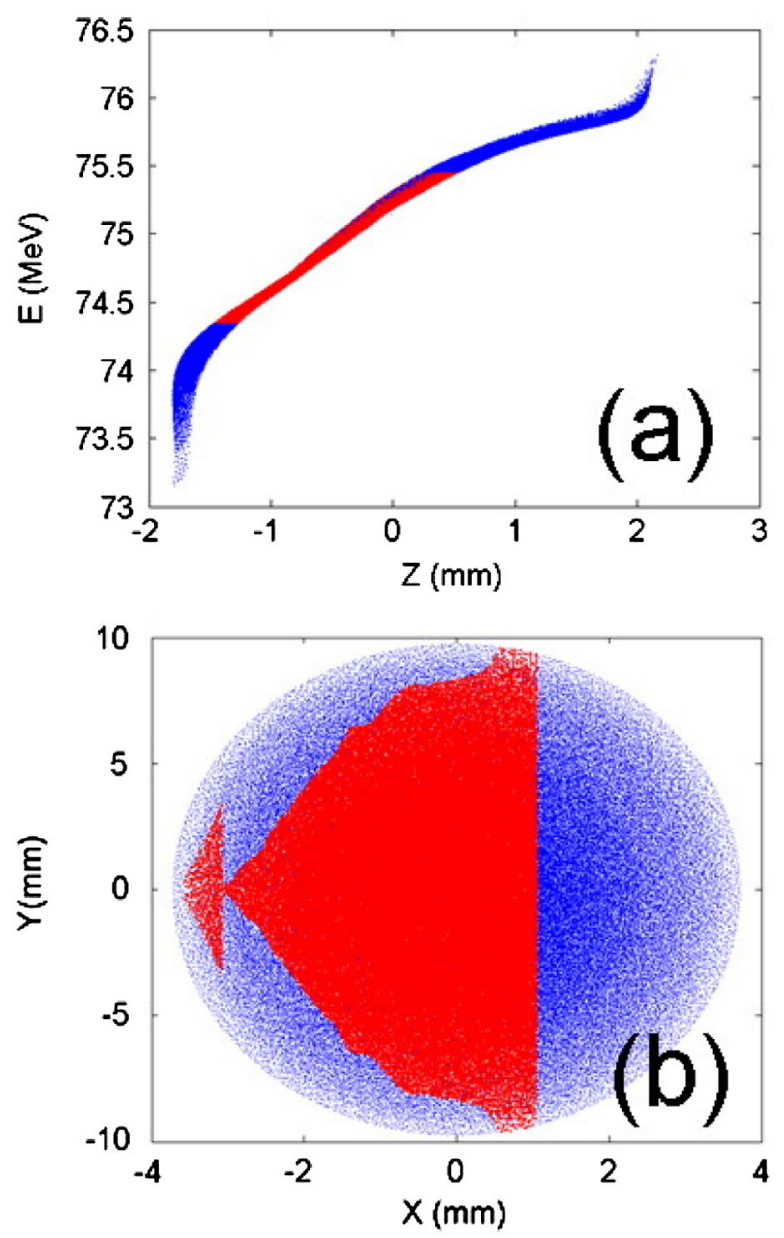

FIG. 4. (a) Longitudinal phase space of the beam after a chicane and energy chopper (red ones remain). (b) Transverse shape of the beam after the transverse mask (red ones remain).

emittance is reduced as well. Both of these conditions favor creating a short bunch after the EEX. Although the mask at the entrance of the EEX beam line reduces the charge from 10 to $\sim 4 \mathrm{nC}$, the shaped bunch can be used in other applications after further acceleration [4]. The effect on the overall efficiency due to the intensity loss at a lower energy can be significantly reduced for a practical accelerating scheme. (For example, we could accelerate the $4 \mathrm{nC}$ shaped bunch to $1 \mathrm{GeV}$ and use it for driving wakefield acceleration. Compared to the loss of $6 \mathrm{nC}$ at $75 \mathrm{MeV}$, there is not a large reduction in overall beam power.)

A chicane is designed at the upstream of the EEX beam line (not shown in Fig. 3) to make the longitudinal phase space $d \delta / d z \approx-1 / \xi$, which reduces the emittance dilution through the EEX beam line. A tunable collimator is installed in the middle of the chicane to chop out diverging particles which may be difficult to control in the EEX. The beam after the chicane and the energy chopper is shown as the blue section of the curve in Fig. 4(a). The beam after passing through the transverse mask is shown in Fig. 4(b). The shape of the mask is optimized for a particular $200 \mathrm{GHz}$ wakefield structure (see Sec. IV for details). At
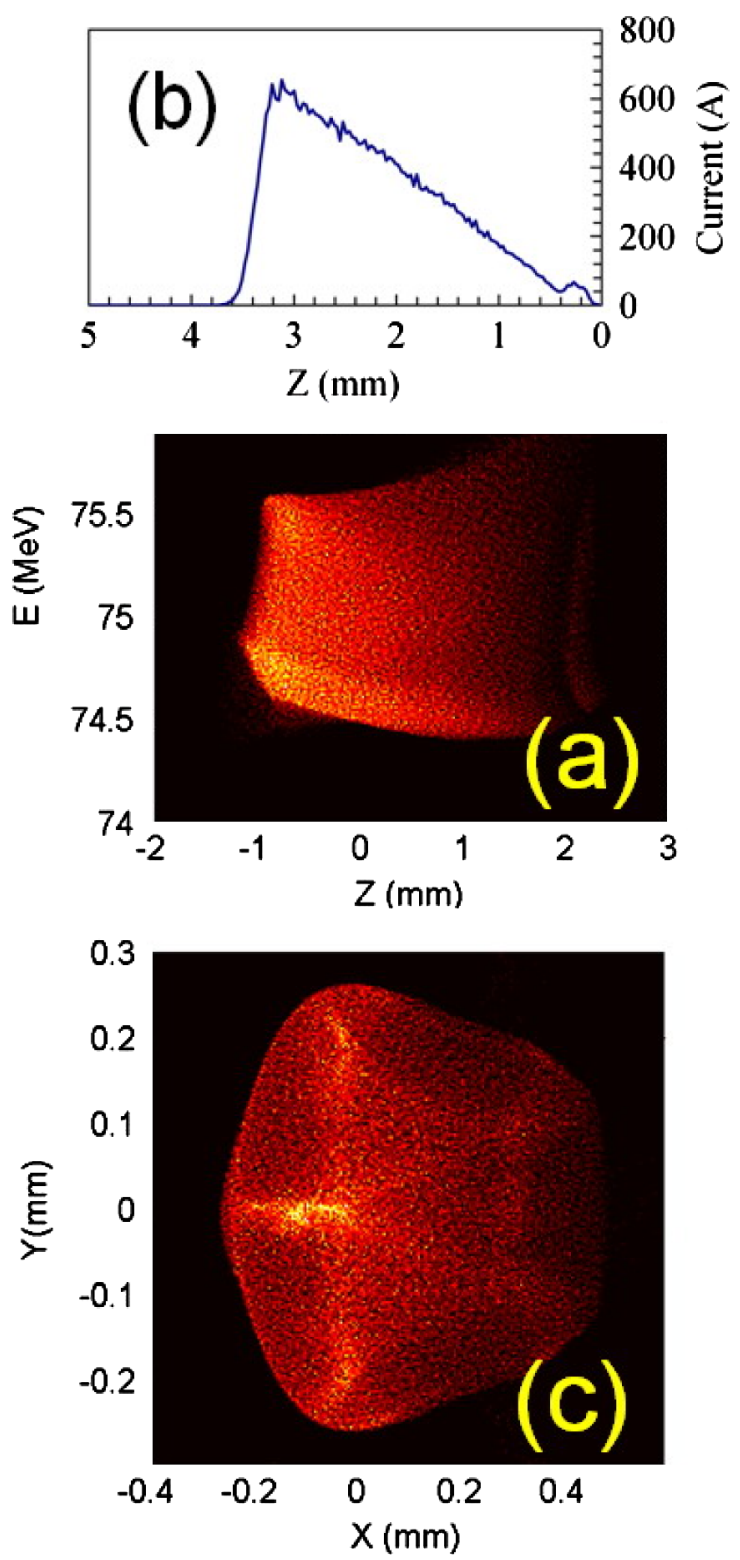

FIG. 5. (a) The simulated longitudinal phase space of the beam at the exit of EEX beam line; (b) beam current profile; (c) transverse size after a quads triplet.

the exit of the EEX beam line, a longitudinally shaped DT bunch is obtained (see Fig. 5). The bunch length is about $3.3 \mathrm{~mm}$. The output beam transverse emittance (normalized) is $74.6 \mathrm{~mm}$ mrad. After the quads the beam size can be focused to $0.6 \mathrm{~mm}$ at the location of the wakefield device under test.

\section{QUASISINGLE MODE COLLINEAR WAKEFIELD ACCELERATOR DESIGN}

Theory suggests that a single mode wakefield device is preferred for maximizing the transformer ratio [9]. Because of its simplicity and low fabrication cost, we plan to use a dielectric wakefield accelerator (DWA) to 
demonstrate the enhanced transformer ratio with the double-triangular bunch. A DWA in its basic configuration is a simple dielectric tube surrounded by a metal jacket. The structure can be characterized with four major parameters: inner radius of the dielectric tube $a$, outer radius $b$, dielectric constant $\varepsilon$, and operating frequency. In general, $a$ is determined by transverse size of the beam. Wakefield strength (without a specific mention we only discuss longitudinal wakefields here) increases with reduction of the inner radius $a$ so that a small $a$ is preferred as long as beam quality allows. (However, a larger value of $a$ is preferred for mitigating the beam loading effect of the witness bunch. A complex tradeoff is required for optimizing the structure.) The frequency spectrum of intrinsic waveguide modes are then fixed once the values of $\varepsilon$ and $b$ are chosen. Waveguide modes are discrete but the mode spectrum has no upper frequency limit (high pass system) while the frequency spectrum (form factor) of a beam is continuous and bandwidth limited (low pass signal). The bandwidth of a beam is determined by the temporal shape of the bunch, for example, the shorter the bunch length the broader the bandwidth. When a beam traverses a wakefield device, only the waveguide modes that overlap with the beam spectrum will be excited. In order to implement single mode operation, we optimize some parameters of the waveguide to remove all high order modes (HOM) from the drive beam bandwidth. For a DWA, this can be done by varying the thickness of the dielectric wall and the dielectric constant (see Fig. 6) while designing the structure. In addition, adjusting these two parameters can also change the loss factor of each mode. We can reduce the loss factor of HOMs so that those modes will be only very weakly excited.

Figure 7 shows the double-triangular bunch and its spectrum that we propose to use in the experiment. As a comparison, a Gaussian bunch is plotted as well. Unlike the Gaussian bunch, we can see that the spectrum of the triangular bunch has a sharper drop at the beginning but then a much slower decrease for frequencies $>\sim 1 / T$. This feature causes difficulties when a single mode wakefield excitation is desired.

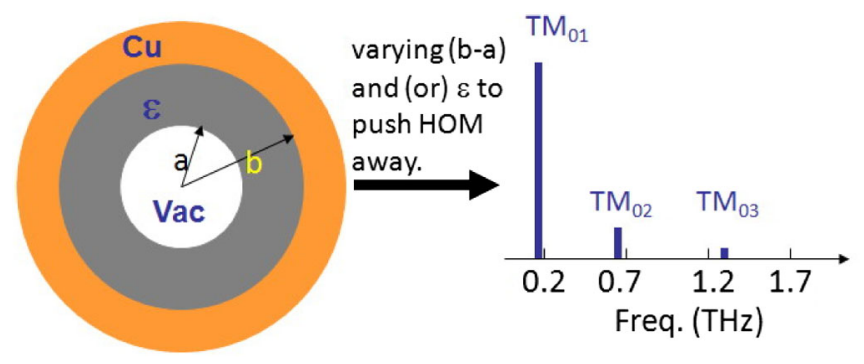

FIG. 6. By changing the parameters of a DWA, the HOMs are pushed out of the bandwidth of the drive beam so that a single mode wakefield excitation is achieved.

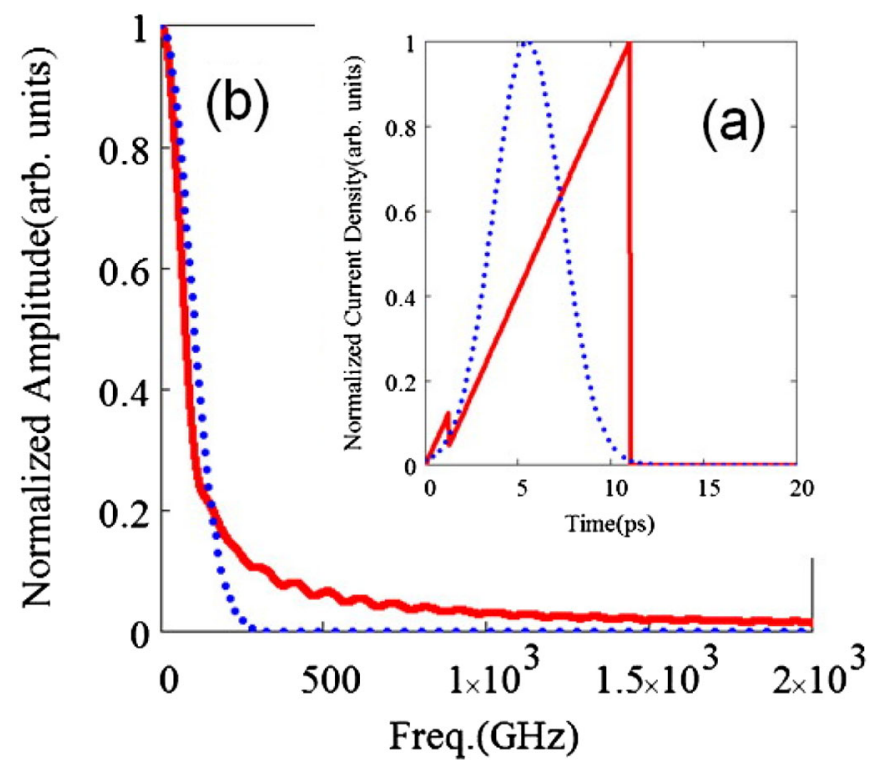

FIG. 7. Comparison of the double-triangular (red solid) and Gaussian (blue dot) bunch with $6 \sigma_{t}=T$, where $\sigma_{t}$ is the bunch length of the Gaussian bunch and $T$ is the total bunch length of the double-triangular bunch: (a) time domain; (b) frequency spectrum.

The goal of the experiment is to demonstrate a high transformer ratio using the specifically shaped electron bunch. Generally speaking, for a fixed average drive current and wakefield structure, the higher the transformer ratio (TR), the lower the wakefield gradient. Our philosophy is to achieve high TR $(R \gg 2)$ while maintaining a moderate gradient so that the energy gain of the witness beam can be easily measured. Let us consider the dependence of the transformer ratio and gradient upon the bunch profile. We first assume that the double-triangular bunch spans the time interval zero to $T$, and $T$ satisfies $T=$ $N^{*} T_{\mathrm{rf}}$, where $T_{\mathrm{rf}}$ is the period of the wakefield behind the bunch and $N$ is a real number. Again, we only consider here the single mode scenario. The average current inside the drive bunch, $I_{\text {ave }}$, can be obtained through integration of its current density distribution described in Eq. (5),

$$
\left(\pi N+\frac{1}{4 N}-1\right) I_{0}=\frac{Q_{\text {total }}}{T}=I_{\text {ave }},
$$

where $Q_{\text {total }}$ represents the total charge of the doubletriangular bunch. Also, the peak wake potential experienced by witness bunch (beam loading of the witness bunch is ignored) and transformer ratio given in Eq. (7) can be simplified as

$$
\left|V^{+}\right|=\frac{I_{0}}{2} \frac{R}{Q} \sqrt{(2 \pi N-1)^{2}+1}
$$

$$
R=\sqrt{1+(2 \pi N-1)^{2}} .
$$



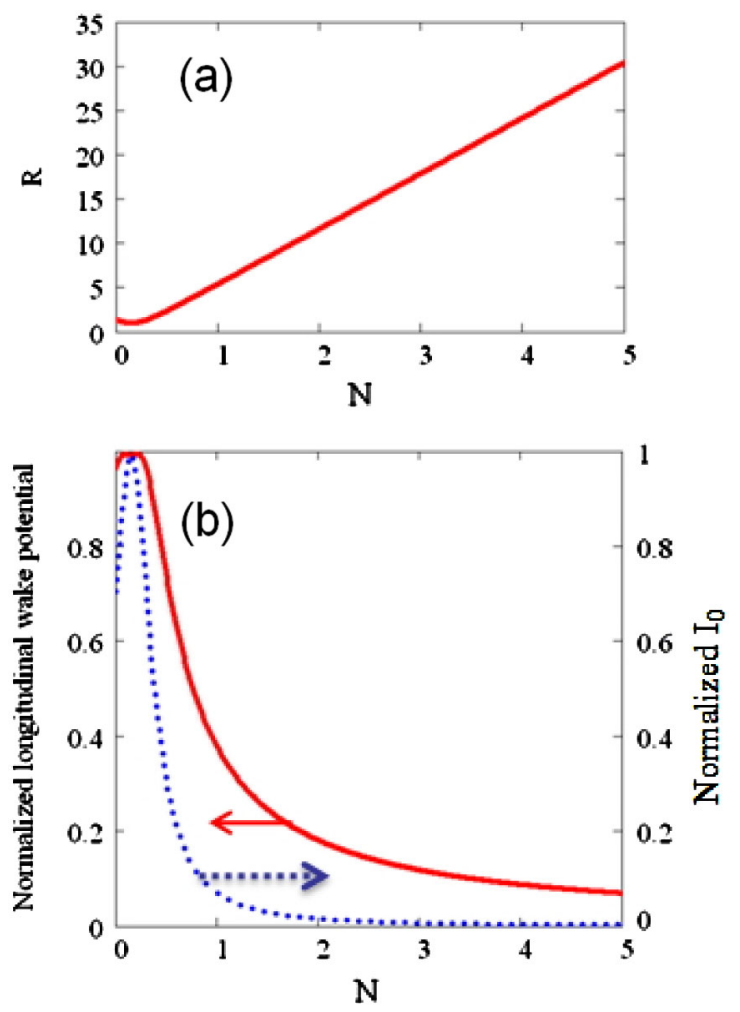

FIG. 8. (a) Plot of Eq. (16): transformer ratio increases as $N$ does; (b) plots of Eqs. (14) and (15): dependences of wake potential (red solid) and the normalization factor $I_{0}$ (blue dot) upon $N$, with the conditions that both $Q_{\text {total }}$ and $R / Q$ are constant.

Once the bunch shape is specified, Eq. (16) clearly shows that $R$ is purely a function of $N$, and is linearly proportional to $N$ as long as $N$ is not very small [Fig. 8(a)]. However, the wakefield gradient has a more complicated behavior with $N$ [Fig. 8(b)]. This is mainly because $I_{0}$ peaks at small $N$ but sharply decreases as $N$ increases, which cancels the positive influence from $N$. Note that drive bunch is fixed, i.e. $I_{\text {ave }}$ is a constant, and we assume that the $R / Q$ of the structure does not change for different frequencies which is generally valid if the radius $a$ of the DWA is fixed.

Considering the simulated temporal profile and transverse size of the drive bunch, we designed a quartz based

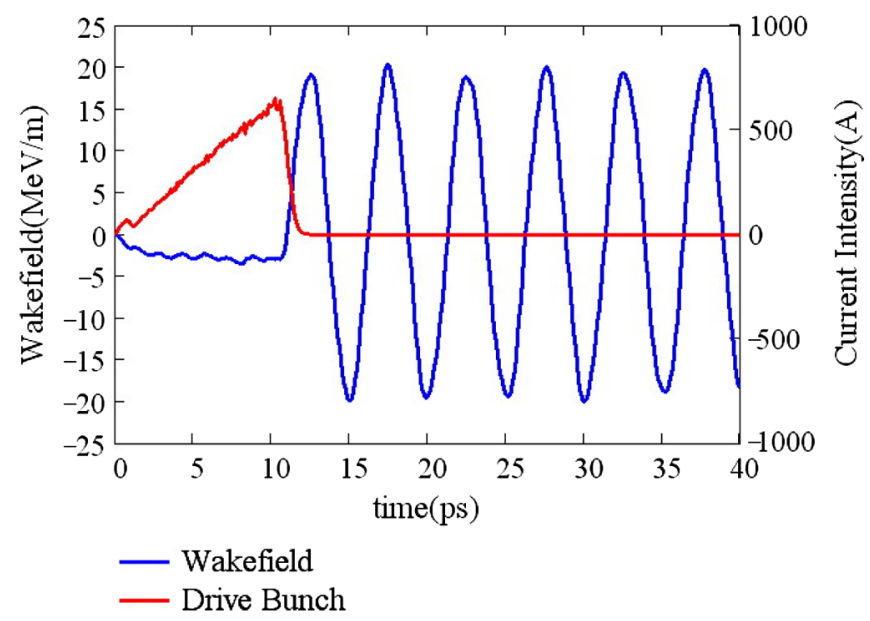

FIG. 9. Current profile of the shaped drive bunch shown in Fig. 5(b) and its excited wakefield in the DWA with parameters shown in Table III.

DWA structure with the beam aperture of $1.5 \mathrm{~mm}$ to perform the high transformer ratio collinear wakefield experiment. The main parameters of the structure are listed in Table III. Instead of a single mode calculation, here a more realistic scenario is considered, i.e., the effects from the high order modes, attenuation factor, and group velocity factor, etc. The material used in the DWA structure is fused quartz so that the fabrication process will benefit from the technology of the optical fiber industry. The calculated wake potential is shown in Fig. 9. The predicted transformer ratio is 6.5 and the gradient is $20 \mathrm{MV} / \mathrm{m}$.

\section{BEAM DYNAMICS IN THE DWA}

The electron phase space as computed by PARMELA at the beginning of the DWA was then sampled to provide input for the calculation of wakefields and beam dynamics in the structure. The BBU3000 code, a $2 \frac{1}{2}$-D particleGreen's function code originally developed by Euclid Techlabs [27] for beam breakup and mitigation studies [28] was used to propagate the beam through the DWA (Fig. 10). Wakefields were calculated up to 4th order in both radial and azimuthal mode numbers (i.e. through $\mathrm{TM}_{04}$ and $\left.\mathrm{HEM}_{44}\right)$.

TABLE III. Main parameters of a dielectric wakefield accelerator to demonstrate a high transformer ratio.

\begin{tabular}{lc}
\hline \hline $\mathrm{ID}, \mathrm{OD}$, length & $1.5 \mathrm{~mm}, 1.8 \mathrm{~mm}, 10 \mathrm{~cm}$ \\
$\varepsilon_{r}, \tan \delta$ & $3.75,6 \times 10^{-4}$ \\
Frequency of $\mathrm{TM}_{01}, \mathrm{TM}_{02}, \mathrm{TM}_{03}, \mathrm{TM}_{04}$ & $198 \mathrm{GHz}, 680 \mathrm{GHz}, 1249 \mathrm{GHz}, 1838 \mathrm{GHz}$ \\
$Q$ of $\mathrm{TM}_{01}, \mathrm{TM}_{02}, \mathrm{TM}_{03}, \mathrm{TM}_{04}$ & $2338,5531,7555,9000$ \\
$r / Q$ of $\mathrm{TM}_{01}, \mathrm{TM}_{02}, \mathrm{TM}_{03}, \mathrm{TM}_{04}$ & $30.4 \mathrm{k} \Omega / \mathrm{m}, 1.4 \mathrm{k} \Omega / \mathrm{m}, 235 \Omega / \mathrm{m}, 74 \Omega / \mathrm{m}$ \\
$V g$ of $\mathrm{TM}_{01}, \mathrm{TM}_{02}, \mathrm{TM}_{03}, \mathrm{TM}_{04}$ & $0.53 \mathrm{c}, 0.75 \mathrm{c}, 0.78 \mathrm{c}, 0.79 \mathrm{c}$ \\
\hline \hline
\end{tabular}




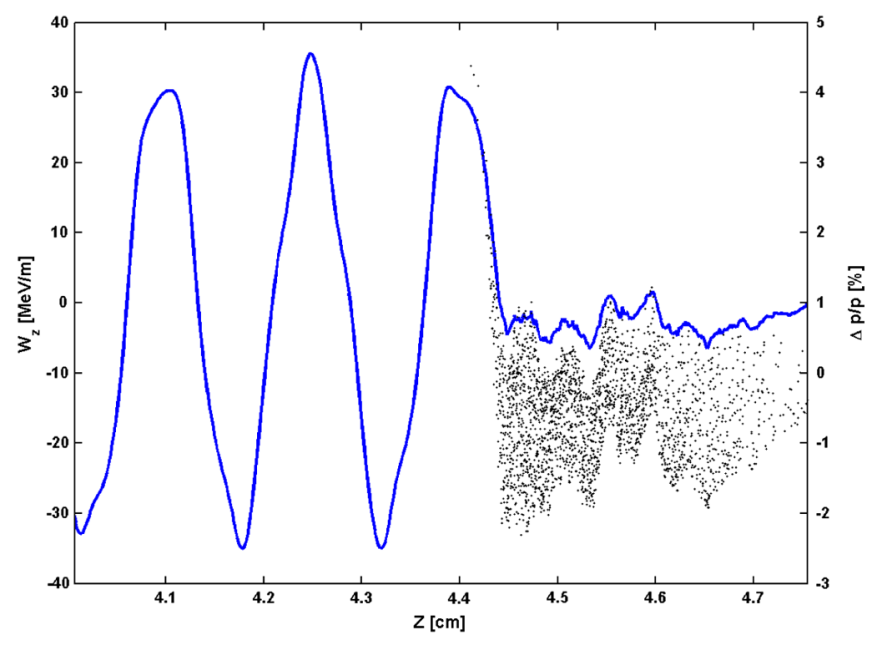

FIG. 10. BBU3000 simulation of the double-triangular bunch experiment. The bunch is passing through a dielectric structure, $a=0.9 \mathrm{~mm}, b=0.75 \mathrm{~mm}$, permittivity $=3.75$. Points: longitudinal phase space (right ordinate); solid line: wake potential (left ordinate). The transformer ratio is $\sim 6.2$.

Figure 10 shows the longitudinal phase space and wakefield of the double-triangular bunch where the beam has propagated about one half of the structure. The transformer ratio computed as (maximum accelerating field behind the bunch)/(peak decelerating field inside the bunch) is about 6.2, in good agreement with the analytic calculations.

As the beam propagates further, particle losses begin to occur from scraping in the vacuum channel of the tube. The beam charge exiting the $10 \mathrm{~cm}$ tube is $\sim 30 \%$ of the initial intensity; the erosion of the bunch reduces the wakefield intensity but does not change the longitudinal current distribution so as to reduce the transformer ratio. The beam transmission may be further improved through more optimized beam optics; alternatively a $5 \mathrm{~cm}$ structure could be used to provide complete transmission.

\section{CONCLUSIONS}

A new asymmetric shaped bunch (double-triangular bunch) to enhance the transformer ratio of wakefield accelerators is analyzed. Using an EEX beam line with a transverse mask at the entrance, the longitudinal bunch current distribution can be tailored in the DT bunch. An experiment has been planned to demonstrate the generation of the shaped bunch and the high transformer ratio in a collinear wakefield acceleration therewith. A full simulation has been completed using the existing EEX beam line at AWA facility. The DWA structure to perform the wakefield acceleration has been constructed. The experiment is scheduled after the new AWA $75 \mathrm{MeV}$ drive beam line is commissioned.

\section{ACKNOWLEDGMENTS}

This work is supported by the U.S. Department of Energy, SBIR office under Grant No. DE-SC0006301.

[1] A. Seryi et al., in Proceedings of the 23rd Particle Accelerator Conference, Vancouver, Canada, 2009 (IEEE, Piscataway, NJ, 2009), pp. 2688-2690.

[2] J. Rosenzweig et al., Nucl. Instrum. Methods Phys. Res., Sect. A 410, 532 (1998).

[3] K. Nakajima, Nature Phys. 4, 92 (2008).

[4] C. Jing et al., in Proceedings of IPAC11, San Sebastián, Spain, 2011, pp. 1485-1487.

[5] K. L. Bane, P. Chen, and P. B. Wilson, IEEE Trans. Nucl. Sci. 32, 3524 (1985).

[6] V. M. Tsakanov, Nucl. Instrum. Methods Phys. Res., Sect. A 432, 202 (1999).

[7] C. Jing et al., Phys. Rev. Lett. 98, 144801 (2007).

[8] C. Jing et al., Phys. Rev. ST Accel. Beams 14, 021302 (2011).

[9] P. Chen et al., Phys. Rev. Lett. 56, 1252 (1986).

[10] R. England, J. Rosenzweig, and G. Travish, Phys. Rev. Lett. 100, 214802 (2008).

[11] M. Cornacchia et al., Phys. Rev. ST Accel. Beams 9, 120701 (2006).

[12] P. Piot et al., in Proceedings of IPAC11, San Sebastián, Spain, 2011, pp. 2805-2807.

[13] P. Piot, Y.-E. Sun, and M. Rihaouic, AIP Conf. Proc. 1086, 677 (2009).

[14] H. Braun et al., Report No. CLIC-Note-764, 2008.

[15] F. Wang et al., in Proceedings of the Advanced Acccelerator Concepts 14th Workshop, 2010, pp. 280-285.

[16] G. V. Sotnikov, T. C. Marshall, and J. L. Hirshfield, Phys. Rev. ST Accel. Beams 12, 061302 (2009).

[17] G. V. Sotnikov et al., AIP Conf. Proc. 1086, 415 (2009).

[18] K. Bane et al., Report No. SLAC-PUB_3662, 1985.

[19] J. Power et al., Phys. Rev. ST Accel. Beams 3, 101302 (2000).

[20] M. Conde et al., in Proceedings of the 11th European Particle Accelerator Conference, Genoa, 2008 (EPS-AG, Genoa, Italy, 2008), pp. 2835-2837.

[21] M. C. Thompson et al., Phys. Rev. Lett. 100, 214801 (2008).

[22] P. Piot et al., Phys. Rev. ST Accel. Beams 14, 022801 (2011).

[23] P. Emma et al., Phys. Rev. ST Accel. Beams 9, 100702 (2006).

[24] Y. -E. Sun et al., in Proceedings of the 2007 Particle Accelerator Conference, Albuquerque, New Mexico (IEEE, New York, 2007), pp. 3441-3443.

[25] www.hep.anl.gov/awa.

[26] J. H. Billen and L. M. Young, PARMELA Reference Manual, Report No. LA-UR-96-1835, 2000.

[27] www.euclidtechlabs.com.

[28] P. Schoessow et al., in Proceedings of the IPAC'10 Conference, Kyoto, Japan (ICR, Kyoto, 2010), pp. 1904-1906. 